\title{
Minimum Discriminants of Imprimitive Decic Fields
}

\author{
Eric D. Driver and John W. Jones
}

\section{CONTENTS}

1. Introduction

2. Type-2 Decics

3. Type-5 Decics

4. Type-2-5 Decics

References
2000 AMS Subject Classification: 11R21, 11Y50, 11R80

Keywords: Number field, global field, minimum discriminiant
In this paper, we compute the minimum discriminants of imprimitive degree-10 fields for different combinations of Galois group and signature. We use class field theory when there is a quintic subfield, and a Martinet search in the more difficult case in which there is only a quadratic subfield.

\section{INTRODUCTION}

If $L$ is a degree- $n$ number field, let $D_{L}$ be its discriminant and $d_{L}:=\left|D_{L}\right|$ its absolute discriminant. Let $G(L)$ be a transitive subgroup of $S_{n}$ giving the Galois group of the Galois closure of $L / \mathbb{Q}$, which is well defined up to conjugation. We let $r_{1}$ denote the number of real places of $L$.

For a given positive integer $n$ and group $G \leq S_{n}$, several authors have contributed to our knowledge of minimizing $d_{L}$ for fields $L$ with $[L: \mathbb{Q}]=n$ and $G(L)=G$. In [Klüners and Malle 01], the authors discuss the more refined problem of minimizing $d_{L}$ with $n, G$, and $r_{1}$ fixed, as well as the still more refined analogue of this question in which the conjugacy class of complex conjugation is specified. Their results are primarily for degrees $\leq 8$, and there have been some results in degree 9 . Here we consider imprimitive fields in degree $n=10$ and attempt to determine minimal examples for fixed $G$ and $r_{1}$.

The computation for imprimitive decics divides naturally into three cases, which we label as follows. We refer to decics $L$ that contain a quadratic but not a quintic subfield as type- 2 fields, those that contain a quintic but no quadratic subfield as type- 5 fields, and those that contain both as type- $2-5$ fields. The most difficult cases are type-2 fields, and here we employed computer searches based on Martinet's generalization of Hunter's theorem. Some of these were standard Martinet searches and some were targeted Martinet searches in the sense of [Driver and Jones 09]. This is explained in Section 2.

Section 3 treats type- 5 decics using class field theory. These computations rely on having a sufficiently large complete list of quintic fields to work from. We extended the range of known totally real quintic fields $K$ from

(c) A K Peters, Ltd 1058-6458/2010 \$0.50 per page Experimental Mathematics 19:3, page 475 
$d_{K} \leq 2 \cdot 10^{7}$ to $d_{K} \leq 10^{8}$, which, in turn, allowed us to determine more minimal decic discriminants.

Finally, in Section 4 we describe results for the simplest case, type-2-5 decic fields. These fields are just tensor products of their subfields, so working with tables of lower-degree fields suffices here. Again, we employed here our extended list of totally real quintic fields.

\section{TYPE-2 DECICS}

\subsection{The Martinet Search}

A standard technique for searching for all fields with absolute discriminant less than a given bound comes from the geometry of numbers. In particular, all such primitive degree- $n$ extensions of a degree- $m$ field can in principle be found using Martinet's theorem, a generalization of Hunter's theorem [Cohen 00, Section 9.3].

Here, we use Martinet's theorem to find all quintic extensions of a quadratic base field. If a decic field $L$ with a quadratic subfield $K$ satisfies the discriminant bound $d_{L} \leq B$, then the discriminant of $K$ must satisfy

$$
d_{K} \leq d_{L}^{1 / 5} \leq B^{1 / 5}
$$

In this paper, we take $B=10^{10}$. Thus, it is necessary to search over each quadratic base field satisfying $d_{K} \leq 100$; there are 61 such quadratic fields.

In [Driver and Jones 09], we show how to target particular discriminants in a Martinet search. Note that our search here is naturally broken up into 61 searches, one for each quadratic field. When the quadratic base field has relatively large discriminant there are correspondingly few possibilities for the decic discriminant beneath the bound $B$. For example, when the quadratic base field is $K=\mathbb{Q}(\sqrt{97})$, the only possibility for the decic field is $d_{L}=97^{5}$.

When we have a priori knowledge of the absolute decic discriminant, we can first test to make sure that the ratio of the absolute polynomial discriminant to $d_{L}$ is a square. This usually weeds out $99 \%$ of the candidate polynomials, and allows us to skip the more computationally expensive tasks of checking for irreducibility and computing field discriminants. Even when multiple discriminants are possible, it is usually much faster to use several targeted searches instead of a single standard search.

The use of targeting is helpful here only for those base fields having the largest discriminant bounds; as the discriminant of the base field drops, a point is reached where too much targeting would be required in order to be practical. In our implementation, we found that a good rule of thumb is to use a targeted Martinet search for those base fields having $d_{K} \geq 0.75 B^{1 / 5}$.

\subsection{Results}

For our first attempt, we used a discriminant bound of $B=10^{9}$. The Martinet search took approximately one week on a 3-GHz Pentium, and found 239 fields.

To extend the search further, we used a distributed computing approach through the BOINC system. ${ }^{1}$ With $B=10^{10}$, the computation took a total of approximately 20,000 machine hours (summed over all hosts). It found 10,565 fields. Table 1 shows the distribution of fields partitioned by type and signature. If no fields of a particular Galois group (respectively signature) were found, then the corresponding row (respectively column) was left out of the table. Impossible combinations of type and signature are grayed out.

Table 2 gives the minimum discriminants for type2 decics as determined by our computer search. Since these polynomials were computationally the most difficult to obtain, we include defining polynomials of the corresponding fields. Here signatures are given by just $r_{1}$, the number of real places. Discriminants are given in factored form. Here and in subsequent tables we include exponents of 1 in a factorization if needed to separate prime factors.

\begin{tabular}{|c|c|c|c|c|}
\hline \multirow{2}{*}{ Galois Group } & \multicolumn{3}{|c|}{ Signature } & \multirow{2}{*}{ Total } \\
\cline { 2 - 4 } & $\mathbf{( 0 , 5 )}$ & $\mathbf{( 2 , 4 )}$ & $\mathbf{( 4 , 3 )}$ & \\
\hline \hline$T 1$ & 1 & & & 1 \\
\hline$T 2$ & 2 & & & 2 \\
\hline$T 3$ & 6 & 2 & & 8 \\
\hline$T 5$ & 1 & 0 & & 1 \\
\hline$T 6$ & 6 & & & 6 \\
\hline$T 11$ & 2 & 0 & & 2 \\
\hline$T 21$ & 14 & 12 & & 26 \\
\hline$T 22$ & 158 & 14 & & 172 \\
\hline$T 33$ & 1 & 0 & & 1 \\
\hline$T 40$ & 2 & 4 & & 6 \\
\hline$T 41$ & 5 & 0 & & 5 \\
\hline$T 43$ & 9187 & 946 & 202 & 10335 \\
\hline
\end{tabular}

TABLE 1. Numbers of decics $L$ with a quadratic subfield and $d_{L} \leq 10^{10}$.

\footnotetext{
${ }^{1}$ Berkeley Open Infrastructure for Network Computing (http: //boinc.berkeley.edu).
} 


\begin{tabular}{|r|r|r|l|}
\hline Galois Group & $\boldsymbol{r}_{\mathbf{1}}$ & Discriminant & Polynomial \\
\hline$T 6$ & 0 & $-3^{5} 31^{4}$ & $x^{10}+2 x^{8}-3 x^{7}+3 x^{6}-7 x^{5}+8 x^{4}-7 x^{3}+7 x^{2}-4 x+1$ \\
\hline$T 21$ & 0 & $-2^{10} 761^{2}$ & $x^{10}-4 x^{9}+8 x^{8}-10 x^{7}+9 x^{6}-6 x^{5}+3 x^{4}-x^{2}+1$ \\
\hline$T 21$ & 2 & $5^{5} 601^{2}$ & $x^{10}-3 x^{9}+5 x^{8}-5 x^{7}+2 x^{6}+2 x^{5}-4 x^{4}+2 x^{3}-2 x+1$ \\
\hline$T 33$ & 0 & $-3^{5} 337^{3}$ & $x^{10}-4 x^{9}+7 x^{8}-11 x^{7}+18 x^{6}-19 x^{5}+16 x^{4}-17 x^{3}+10 x^{2}-3 x+3$ \\
\hline$T 40$ & 0 & $-3^{5} 4903^{2}$ & $x^{10}-x^{9}+5 x^{8}-3 x^{7}+8 x^{6}-4 x^{5}+4 x^{4}-3 x^{3}-x+1$ \\
\hline$T 40$ & 2 & $5^{5} 1429^{2}$ & $x^{10}-4 x^{9}+8 x^{8}-12 x^{7}+18 x^{6}-21 x^{5}+18 x^{4}-9 x^{3}+x^{2}+2 x-1$ \\
\hline$T 41$ & 0 & $-2^{6} 3^{5} 13^{2} 43^{2}$ & $x^{10}-x^{9}-4 x^{8}+x^{7}+8 x^{6}+2 x^{5}-6 x^{4}-x^{3}+7 x^{2}+5 x+1$ \\
\hline$T 43$ & 0 & $-3^{5} 11^{2} 7369$ & $x^{10}-3 x^{9}+5 x^{8}-6 x^{7}+6 x^{6}-4 x^{5}+2 x^{4}-x^{3}+x^{2}-x+1$ \\
\hline$T 43$ & 2 & $5^{5} 280001$ & $x^{10}-x^{9}-x^{8}+3 x^{7}-3 x^{6}-x^{5}+5 x^{4}-x^{3}-3 x^{2}+x+1$ \\
\hline$T 43$ & 4 & $-5^{5} 1039^{1} 1049$ & $x^{10}-3 x^{9}+5 x^{8}-4 x^{7}-2 x^{6}+8 x^{5}-8 x^{4}+x^{3}+3 x^{2}-3 x+1$ \\
\hline
\end{tabular}

TABLE 2. Minimum discriminants and defining polynomials of type- 2 decics.

\section{TYPE-5-DECICS}

\subsection{Totally Real Quintics}

Studying type-5 decics relies on our knowledge of quintic fields. In the case of totally real quintics $K$, those with $d_{K} \leq 2 \cdot 10^{7}$ were classified by [Schwarz et al. 94], and are available at the Bordeaux FTP site. ${ }^{2}$ While the bound for $d_{K}$ is large compared to the corresponding bounds for the two other signatures of quintic fields, it accounts for only 22,740 fields, which we found insufficient.

We implemented a standard Hunter's search using the methods described in [Cohen 00, Section 9.3] to find all totally real quintic fields $K$ with $d_{K} \leq 10^{8}$. The number of fields broken down by Galois group is given in Table 3 .

Here we use common names for the Galois groups, which correspond to $T 1-T 5$ respectively using the labeling of [Butler and McKay 83]. ${ }^{3}$ As was the case in [Schwarz et al. 94], almost all of the fields have Galois group $S_{5}$. Although this is a fairly modest increase in the range of discriminants, it was of great use in computing the minimal absolute discriminants of totally real decics of type 5 and type $2-5$.

\begin{tabular}{|c|c|c|c|c|c|}
\hline Galois Group & $C_{5}$ & $D_{5}$ & $F_{5}$ & $A_{5}$ & $S_{5}$ \\
\hline Number of Fields & 6 & 72 & 59 & 80 & 162,022 \\
\hline
\end{tabular}

TABLE 3. Numbers of totally real quintic number fields $K$ with $d_{K} \leq 10^{8}$, partitioned by Galois group.

\footnotetext{
${ }^{2}$ The Bordeaux Database of Number Fields (ftp://megrez.math. u-bordeaux.fr/pub/numberfields).

${ }^{3}$ The fields themselves are available online: "Tables of Number Fields with Prescribed Ramification" (http://math.la.asu.edu/ jj/ numberfields).
}

\subsection{Class Field Theory}

For decic fields containing a quintic subfield, class field theory provides a mechanism for computing the desired fields. Here, computations are relatively simple in comparison to more general cases of Kummer theory, since our fields always contain $\zeta_{2}=-1$, so the desired extensions can be found by taking square roots of appropriate elements. An algorithm for this is given in [Cohen 00, Section 9.2.2].

All computations here were carried out with gp. ${ }^{4}$ We needed to deviate from the approach given in [Cohen 00] because of memory issues. Given a base field $K$ and bound $B$, a quadratic extension corresponding to modulus $\left(\mathfrak{m}_{0}, \mathfrak{m}_{\infty}\right)$ needs to satisfy $d_{K}^{2} N\left(\mathfrak{m}_{0}\right) \leq B$. In some cases, it was impractical to first compute all $\mathfrak{m}_{0}$ with $N\left(\mathfrak{m}_{0}\right) \leq B / d_{K}^{2}$. Instead, we computed the list $\mathcal{L}$ of all such moduli that are divisible only by primes $p \leq B_{1}:=$ $\sqrt{B / d_{K}^{2}}$. All additional moduli are then of the form $\mathfrak{m}_{0} Q$, where $Q$ divides a prime $q$ with $B_{1}<q \leq B / d_{K}^{2}$ and $\mathfrak{m}_{0}$ is from $\mathcal{L}$. So as we compute these, we compute the corresponding fields as we go and do not have to store these additional moduli.

For example, consider the totally real $S_{5}$ quintic field of minimal discriminant. It has discriminant $61 \cdot 397$. With $B=10^{16}$, the first phase found and stored the 766,157 moduli that are divisible only by primes less than $B_{1}=4129$. The total number of moduli was $3,851,600$.

One could compute decic overfields of quintics using a Martinet search, as was done in [Selmane 00a, Selmane 00b, Selmane 02]. We found, however, that class field theory was much more efficient.

\footnotetext{
${ }^{4}$ Available online: PARI/GP, version 2.3.3, Bordeaux, 2008, http://pari.math.u-bordeaux.fr/.
} 


\begin{tabular}{|r|c||r|r|r|r|r|r|}
\hline $\boldsymbol{r}_{\mathbf{1}}$ & $\boldsymbol{B}$ & \multicolumn{1}{c|}{0} & \multicolumn{1}{c|}{2} & 4 & 6 & 8 & 10 \\
\hline \hline 1 & $10^{12}$ & 226764 & 210453 & & & & \\
\hline 3 & $10^{12}$ & 11972 & 37249 & 32852 & 7876 & & \\
\hline 5 & $10^{13}$ & 772 & 3954 & 7527 & 7592 & 2849 & 313 \\
\hline
\end{tabular}

TABLE 4. Numbers of type- 5 decic fields $L$ with $d_{L} \leq B$ by signature. Columns correspond to the number of real places for the decic field, and rows correspond to the number of real places of the quintic subfield.

\begin{tabular}{|c|c|c|c|c|c|c|}
\hline \multirow{2}{*}{ Gal } & \multicolumn{5}{|c|}{ Signature } \\
\cline { 2 - 7 } & $(0,5)$ & $(2,4)$ & $(4,3)$ & $(6,2)$ & $(8,1)$ & $(10,0)$ \\
\hline \hline$T 8$ & & $11^{8} 23^{2}$ & & $11^{8} 23^{2}$ & & $2^{10} 11^{8} 23^{2}$ \\
\hline$T 14$ & $-11^{8} 1451$ & $11^{8} 89$ & $-11^{8} 23$ & $11^{8} 109$ & $-11^{8} 331$ & $11^{8} 43^{1} 67$ \\
\hline$T 15$ & & $2^{10} 47^{4}$ & & $3^{2} 401^{4}$ & & $67^{2} 410^{4}$ \\
\hline$T 16$ & $-5^{2} 47^{5}$ & $3^{2} 401^{5}$ & & $401^{5}$ & & $17^{2} 401^{5}$ \\
\hline$T 23$ & $-47^{4} 83$ & $47^{4} 193$ & $-3^{1} 401^{4}$ & $13^{1} 401^{4}$ & $-3^{3} 401^{4}$ & $397^{1} 401^{4}$ \\
\hline T24 & & $2^{16} 13^{6}$ & & $2^{10} 5^{10} 7^{4}$ & & $?$ \\
\hline T25 & & $2^{12} 13^{7}$ & & $2^{10} 5^{11} 7^{4}$ & & $?$ \\
\hline T29 & $-2^{8} 13^{6} 31$ & $2^{8} 13^{6} 41$ & $-2^{8} 3^{1} 53^{6}$ & $2^{8} 5^{1} 53^{6}$ & $-2^{8} 19^{1} 53^{6}$ & $2^{8} 5^{10} 7^{4} 97$ \\
\hline T34 & & $?$ & & $3^{4} 883^{4}$ & & $2^{6} 11^{4} 113^{4}$ \\
\hline T36 & $-2^{12} 17^{4} 43$ & $2^{14} 11^{1} 17^{4}$ & $-2^{4} 3^{1} 881^{4}$ & $2^{6} 17^{1} 881^{4}$ & $-3^{5} 883^{4}$ & $2^{4} 11^{4} 29^{1} 113^{4}$ \\
\hline T37 & & $5^{2} 5783^{2}$ & & $2^{10} 13^{2} 347^{2}$ & & $2^{10} 36497^{2}$ \\
\hline T38 & $-3^{8} 223^{3}$ & $2^{8} 227^{3}$ & $-2^{8} 13^{4} 19^{3}$ & $61^{3} 397^{3}$ & & $2^{4} 33769^{3}$ \\
\hline T39 & $-7^{2} 23^{1} 431^{2}$ & $7^{2} 41^{1} 631^{2}$ & $-7^{2} 43^{1} 1217^{2}$ & $461^{1} 5783^{2}$ & $-61^{2} 167^{1} 397^{2}$ & $61^{2} 397^{2} 757$ \\
\hline
\end{tabular}

TABLE 5. Minimum discriminants for type-5 decics.

\subsection{Results}

For complete searches of type- 5 decics, we computed all fields $L$ with $d_{L} \leq 10^{12}$. When the quintic subfield was totally real, we computed all decics with $d_{L} \leq 10^{13}$. The results are summarized in Table 4.

These computations include, and extend, those performed in [Selmane 00a, Selmane 00b, Selmane 02]. We found one discrepancy with these prior computations; namely, we found one more totally complex decic field with a quintic subfield with $r_{1}=3$ and $d_{L} \leq 10^{11}$. Otherwise, our results are consistent with Selmane's.

Table 5 gives our findings for minimal absolute discriminants for type- 5 decics. Most entries come from the searches summarized above, namely from fields with $d_{L} \leq 10^{12}$. Almost all the cases not settled by that search had a totally real quintic subfield. This naturally includes totally real decics $\left(r_{1}=10\right)$, but also cases in which $\left(T, r_{1}\right)=(16,2),(16,6),(24,6),(25,6),(29,4)$, $(29,6),(29,8),(34,6),(36,4),(36,6),(36,8),(38,6)$.
For example, consider a $T 16$ decic $L$ with $r_{2}=2$. The group $T 16$ has two conjugacy classes of elements of order 2 and cycle type $2^{2} 1^{6}$. The unique quintic subfield $K$ has $G(K)=D_{5}$, and in the projection $T 16 \rightarrow D_{5}$ induced by the inclusion $K \hookrightarrow L$, elements of cycle type $2^{2} 1^{6}$ map to the identity. So, for a $T 16$ decic with $r_{1}=2$, the quintic subfield is a totally real $D_{5}$ field.

In these cases, we made use of the quintics computed in Section 3.1. In each case, we computed decic overfields of the desired signature, where the quintic had the correct Galois group, and $d_{L} \leq 10^{16}$. In this computation, we kept track only of fields of minimal discriminant for each Galois group, since the number of fields in some cases was so large.

\section{TYPE-2-5 DECICS}

The simplest case is that in which the decic contains both a quadratic and quintic subfield. There are eight corresponding decic Galois groups. These fields are easily 


\begin{tabular}{|c|c|c|c|c|}
\hline \multirow{2}{*}{ Gal } & \multicolumn{4}{|c|}{ Signature } \\
\cline { 2 - 4 } & $(0,5)$ & $(2,4)$ & $(6,2)$ & $(10,0)$ \\
\hline \hline$T 1$ & $-11^{9}$ & & & $3^{5} 11^{9}$ \\
\hline$T 2$ & $-47^{5}$ & & & $401^{5}$ \\
\hline$T 3$ & $-3^{5} 47^{4}$ & $7^{4} 17^{5}$ & & $5^{5} 401^{4}$ \\
\hline$T 4$ & & $2^{8} 5^{11}$ & & $2^{8} 5^{11} 7^{4}$ \\
\hline$T 5$ & $-3^{5} 17^{6}$ & $2^{15} 5^{10}$ & & $2^{15} 7^{4} 13^{6}$ \\
\hline$T 11$ & $-2^{16} 17^{4}$ & $5^{5} 53^{4}$ & & $3^{8} 5^{5} 73^{4}$ \\
\hline$T 12$ & $-2^{10} 89^{4}$ & $2^{8} 3^{8} 5^{7}$ & & $2^{8} 13^{7} 17^{4}$ \\
\hline$T 22$ & $-3^{5} 1123^{2}$ & $5^{5} 13^{2} 41^{2}$ & $13^{5} 347^{2}$ & $5^{5} 61^{2} 397^{2}$ \\
\hline
\end{tabular}

TABLE 6. Minimum discriminants of Type- $2-5$ decics.

generated from tables of quadratics and quintics by forming composita. We used quintics from the Bordeaux FTP site augmented by the results of our search for totally real quintics described in Section 3.1 above. Naturally, some care must be exercised to ensure that one has searched far enough. Using this method, we were able to find the minimum discriminants for all the above-mentioned decics, and for almost all possible signatures, with the results given in Table 6 .

\section{REFERENCES}

[Butler and McKay 83] G. Butler and J. McKay. "The Transitive Groups of Degree up to 11." Comm. Algebra 11 (1983), 863-911.

[Cohen 00] H. Cohen. Advanced Topics in Computational Number Theory. New York: Springer, 2000.

[Driver and Jones 09] E. Driver and J. Jones. "A Targeted Martinet Search." Math. Comp. 78 (2009), 1109-1117.

[Klüners and Malle 01] J. Klüners and G. Malle. "A Database for Field Extensions of the Rationals." LMS J. Comput. Math. 4 (2001), 182-196.

[Schwarz et al. 94] A. Schwarz, M. Pohst, and F. Diaz y Diaz. "A Table of Quintic Number Fields." Math. Comp. 63 (1994), 361-376.

[Selmane 00a] S. Selmane. "Quadratic Extensions of Totally Real Quintic Fields." Math. Comp. 70 (2000), 837-843.

[Selmane 00b] S. Selmane. "Tenth Degree Number Fields with Quintic Fields Having One Real Place." Math. Comp. 70 (2000), 845-851.

[Selmane 02] S. Selmane. "Quadratic Extensions of Quintic Fields of Signature $(3,1)$." Acta Math. Inform. Univ. Ostraviensis 10:1 (2002), 117-123.

Eric D. Driver, Lockheed Martin, 4318 N. 69th Place, Scottsdale, AZ 85251 (eric.driver@lmco.com)

John W. Jones, School of Mathematical and Statistical Sciences, Arizona State University, Box 871804, Tempe, AZ 85287 (jj@asu.edu)

Received September 1, 2009; accepted September 29, 2009. 